\title{
Pole-to-pole ocean viromes
}

\author{
A global-scale survey of the ocean viral metagenome (virome) broadens \\ views on marine viruses and their roles in ecosystems.
}

n oceans, microorganisms including bacteria, archaea, and viruses represent major players in marine biodiversity for maintaining essential ecosystem functions. Among the microorganisms, viruses not only have a role in bacterial mortality, but also influence microbial evolution through gene transfer and metabolic reprogramming. These genome-level effects on bacteria prompt a foundational question on how viruses take part in gene-to-ecosystem models.

Global surveys of ocean microbes have just begun to emerge. Yet studies on the ocean viral community's impact on the ecosystem are still hampered by the limited understanding of viral genome landscapes and the lack of methods for global-scale comparative analyses.

To explore the ocean viral communities at the genome level, an international team has sequenced 145 samples collected across the world's oceans and created a virome roadmap, Global Ocean Viromes (GOV) 2.0. They expanded the original version (GOV 1.0) by more deeply sequencing past samples, as well as including 41 new samples collected from the Tara Oceans Polar Circle expedition around the Arctic Ocean in 2013, which supplemented the virome roadmap with representations from extreme environments.

Matthew Sullivan, a microbiologist at the Ohio State University and also a scientific coordinator of the Tara Oceans Consortium, comments that "it has long been a dream to develop better 'maps' of viruses globally ... and the Tara Oceans Consortium made that dream a possibility." Sullivan has had a longstanding interest in ocean viruses since he started his PhD in 1998, for which he studied photosynthetic cyanobacteria and their viruses. He notes that "these cells are tiny, but they are responsible for about one in six breaths' worth of oxygen that you take, as they are abundant and distributed near-globally."

The surveyed 145 samples of viral communities uncovered nearly 200,000 marine viral populations, which largely exceeds the original GOV dataset. The viral communities could be relatively cleanly separated into five ecological zones, globally: bathypelagic, temperate and tropical epipelagic, mesopelagic, and two Arctic regions. Nevertheless, it is not straightforward to explore the changes in diversity across ecological zones. To this end, Sullivan lab members and leaders of this research Ann Gregory and Ahmed Zayed calculated two levels of diversity: macrodiversity, which reflects the diversity of populations within one community, and microdiversity, which represents the diversity of genetic variations within populations.

The diversity analyses revealed that both latitudinal gradients and vertical layers of the ocean have substantial effects on both macroand micro-levels. It is known that the species diversity of larger organisms is low near the poles compared with that close to the equator. However, Sullivan comments, "we were most surprised by the Arctic biodiversity hot spot emerging, and that has strong implications for climate change feedbacks, since these are rapidly impacted ecosystems."

The meta-community analyses were feasible only with computational tools that are capable of handling large global virome datasets. Sullivan and collaborators have developed iVirus as a community resource for viromic tools and datasets. To facilitate viral classification and taxonomy, they offer version 2.0 of vConTACT for handling large global virome datasets. "A key deliverable are features like the ability to easily identify and capture gene markers within subsets of the network to do targeted studies on those viruses."

With the updated global map and tools, researchers now have the opportunity to better understand the features of abundant viral populations and their roles in modulating microbial metabolisms through a genome-centered approach.

Lei Tang

Published online: 27 June 2019

https://doi.org/10.1038/s41592-019-0480-1

Research papers

Gregory, A. C. et al. Marine DNA viral macroand microdiversity from pole to pole. Cell 177, 1109-1123 (2019).

Jang, H. B. et al. Taxonomic assignment of uncultivated prokaryotic virus genomes is enabled by gene-sharing networks. Nat. Biotechnol. 37, 632-639 (2019).

Bolduc, B. et al. iVirus: facilitating new insights in viral ecology with software and community data sets imbedded in a cyberinfrastructure. ISME J. 11 7-14 (2017). nature MASTERCLASSES

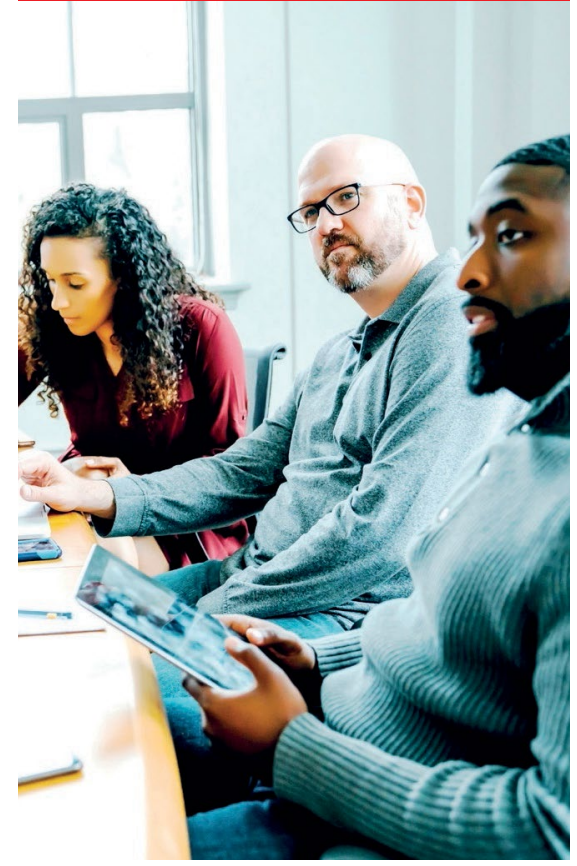

Workshops in

Scientific Writing

and Publishing

Delivered by Nature Research

journal editors and hosted

at institutions worldwide,

researchers learn how to

turn great science into great

papers.

Find out more at

masterclasses.nature.com

W masterclasses.nature.com

in Follow us on Linkedln 